\title{
Ethylene-triggered subcellular trafficking of CTR1 suppresses the response to ethylene
} gas.

Han Yong Lee ${ }^{1,2, \dagger}$, Dong Hye Seo ${ }^{1,2, ¥ ¥}$, Arkadipta Bakshi” ${ }^{3 \#}$, Hye Lin Park ${ }^{1,2}$, Chanung Park ${ }^{1,2}$, Joseph J. Kieber ${ }^{4}$, Brad M. Binder ${ }^{3}$, and Gyeong Mee Yoon ${ }^{1,2, *}$.

${ }^{1}$ Department of Botany and Plant Pathology, Purdue University, West Lafayette, Indiana, 47907, USA.

${ }^{2}$ Center for Plant Biology, Purdue University, West Lafayette, Indiana, 47907, USA.

${ }^{3}$ Department of Biochemistry \& Cellular and Molecular Biology, University of Tennessee, Knoxville, Tennessee, 37996, USA.

${ }^{4}$ Department of Biology, University of North Carolina, Chapel Hill, North Carolina, 27599, USA.

\# Current address: Department of Botany, UW-Madison, Madison, Wisconsin, USA.

¥ Current address: Department of Systems Biology, Yonsei University, Seoul, 03722, Korea.

*Correspondence: yoong@purdue.edu

${ }^{\dagger} H Y$. Lee and DH. Seo contributed equally to this work. 


\section{Summary:}

Ethylene gas controls plant growth and stress responses. Ethylene-exposed dark-grown seedlings exhibit a dramatic growth reduction, yet the seedlings rapidly return to the basal growth rate when ethylene gas is removed. However, the underlying mechanism governing this remarkable reversible acclimation of dark-grown seedlings to ethylene remains enigmatic. Here, we report that ethylene triggers the translocation of the Raf-like protein kinase CONSTITUTIVE TRIPLE RESPONSE1 (CTR1), a negative regulator of ethylene signaling, from the endoplasmic reticulum to the nucleus. Nuclear-localized CTR1 inhibits the ETHYLENE-INSENSITIVE3 (EIN3) transcription factor via the EIN3-BINDING F-box Proteins, resulting in rapid suppression of the ethylene response, thus promoting fast growth recovery. These findings uncover a mechanism of the ethylene signaling pathway that links the spatiotemporal dynamics of cellular signaling components to organismal responses.

Key Words: Ethylene, CTR1, Nuclear localization, Spatiotemporal regulation, Growth Kinetics, Stress response 


\section{Introduction:}

The ability of organisms to respond to and integrate environmental signals leading to an appropriate response is critical for optimal growth and development, particularly for plants, which are non-motile. Plants adapt to a wide variety of abiotic stresses, and upon removal of stress, they need to rapidly restore basal cellular homeostasis. One key signal for abiotic stress is the plant hormone ethylene. Ethylene is involved in multiple aspects of growth and development, including fruit ripening, leaf and floral senescence, cell elongation, seed germination, and root hair formation, as well as responses to biotic and abiotic stress ${ }^{1-3}$. Ethylene-mediated stress acclimation includes, but is not limited to, the rapid elongation of rice internodes in response to flooding, drought responses, salt tolerance, heavy metal tolerance, and morphological changes of roots in response to nutrient deficiency ${ }^{4-8}$. How ethylene regulates such remarkable plasticity of plant stress adaptation is poorly understood, however.

Extensive molecular genetic studies have elucidated the basic ethylene signaling pathway $^{2,9-12}$. In the absence of ethylene, the endoplasmic reticulum (ER)-localized ethylene receptors activate the CTR1 protein kinase, which in turn phosphorylates Ethylene-Insensitive 2 (EIN2), an ER membrane-localized Nramp homolog that positively regulates ethylene responses. CTR1-mediated phosphorylation of EIN2 leads to its ubiquitination and proteolysis by the $26 \mathrm{~S}$ proteasome ${ }^{11-18}$. In response to ethylene, the receptors, and hence CTR1, are inactivated, leading to reduced phosphorylation and increased accumulation of EIN2. EIN2 is then proteolytically cleaved, releasing the C-terminal domain (EIN2-CEND) which translocates into the nucleus where it activates the Ethylene-Insensitive 3 (EIN3) and EIN3-Like (EIL) paralogs which function as central transcription factors in ethylene signaling ${ }^{16-18}$. EIN2-CEND also associates with the EIN3-Binding F-box 1 (EBF1) and EBF2 mRNAs and represses their translation, thus blocking the degradation of EIN3/EIL proteins ${ }^{19,20}$. The function of CTR1 beyond its phosphorylation of EIN2 at the ER, if any, has not been characterized. 
The hypocotyls of dark-grown seedlings exposed to ethylene show a dramatic reduction in growth rate. However, following the removal of ethylene, the seedlings return to a basal rate of growth within $90 \min ^{21,22}$, even though the proteolytic cleavage of EIN2 is irreversible. How the nuclear-localized EIN2-CEND is turned off in the nucleus is an open question. Here, we report that CTR1 rapidly translocates from the ER to the nucleus in response to ethylene. The nuclear-localized CTR1 inhibits ethylene responses through a mechanism that does not require its kinase activity and promotes the fast recovery of seedling growth back to basal levels. These results suggest a new mechanistic paradigm for the dynamic regulation of the ethylene signaling involving the translocation of CTR1 to the nucleus, which counteracts EIN2-mediated EIN3 10 activation in the nucleus.

\section{Results:}

\section{Ethylene-induced ER-to-nucleus translocation of CTR1.}

CTR1 consists of an $\mathrm{N}$-terminal regulatory domain and a $\mathrm{C}$-terminal kinase domain that is homologous to the catalytic domain of the Raf kinase family ${ }^{11}$ (Fig.1a). To determine the role of CTR1 beyond its role in regulating EIN2, we examined the subcellular localization of CTR1 following exposure to exogenous ethylene in the dark. To this end, we introduced a $\sim 7.6 \mathrm{~Kb}$ genomic CTR1 transgene that contains the CTR1 promoter region $(0.7 \mathrm{~K} \mathrm{~b}$ upstream of the 5'UTR) driving expression of the CTR1 coding region fused to a GFP reporter (CTR1p:GFPgCTR1) into the ctr1-2 loss-of-function mutant ${ }^{11}$. The CTR1p:GFP-gCTR1 transgene fully complemented the ctr1-2 phenotypes in both light and dark-grown seedlings (Fig.1b and Supplementary Fig.1), indicating that this fusion protein is functional. In the absence of ethylene signaling which was generated by the treatment of seedlings with silver nitrate $\left(\mathrm{AgNO}_{3}\right)$, an ethylene perception inhibitor, the GFP-CTR1 fusion protein localized to the ER (Fig.1c), consistent with previous reports ${ }^{14,15}$. However, surprisingly, in response to either 1 aminocyclopropane-1-carboxylate (ACC), a direct precursor of ethylene, or exogenous 
ethylene, GFP-CTR1 accumulated in the nucleus (Fig.1c, 1d, and 1e). In contrast to etiolated seedlings, GFP-CTR1 constitutively localized to the nucleus in light-grown seedlings (Fig. 1f). This may be due to higher basal ethylene levels in light-grown seedlings (Fig.1g) ${ }^{23}$. However, it is also likely that an ethylene-independent pathway can also stimulate CTR1 nuclear localization in light since silver thiosulfate (STS), an ethylene perception inhibitor, did not prevent the nuclear movement of CTR1 (Fig.1f). Furthermore, when GFP-CTR1 was expressed under the control of the strong CaMV 35S promoter, the large fraction of GFP-CTR1 constitutively localized to the nucleus in etiolated seedlings, regardless of ACC treatment (Fig.1h), presumably due to a limited amount of ethylene receptors tethering CTR1 to the ER. As both ACC and ethylene caused equivalent CTR1 nuclear translocation (Fig.1e), we employed ACC for further studies. Disruption of either EIN2 or EIN3/EIL1 did not prevent the nuclear accumulation of CTR1 (Fig.1I), suggesting that EIN2 and EIN3 are not required for CTR1 nuclear translocation.

EIN2-CEND migrates into the nucleus within 10 min following ethylene treatment ${ }^{17}$. To determine the nuclear movement kinetics of CTR1 upon ethylene perception, we monitored the dynamics of the CTR1 movement in response to ACC treatment. CTR 1 first began to accumulate in the nucleus 30 min after ACC treatment, with a further increase in nuclear protein levels after an additional $30 \mathrm{~min}$ (Fig.1J). Overall levels of GFP fluorescence intensity were enhanced with longer ACC exposure, consistent with increased stabilization of CTR1 protein in response to ACC (Fig.1K), similar to previous reports ${ }^{24}$. Together, these results showed that ethylene stimulates the translocation of CTR1 from the ER to the nucleus in an EIN2 and EIN3/EIL independent manner.

\section{The CTR1 N-terminus inhibits CTR1 nuclear trafficking.} Ethylene receptors are transmembrane proteins, thus nuclear translocation of CTR1 must involve dissociation of CTR1 from the receptors. Since CTR1 interacts with the ETR1 ethylene 
receptor via its $\mathrm{N}$-terminal domain ${ }^{14}$, we examined whether the $\mathrm{N}$-terminal domain of CTR1 affects CTR1 nuclear translocation. To test this, N-terminal domain-deleted CTR1 protein harboring only the kinase domain ( $\triangle \mathrm{NT}-\mathrm{CTR} 1)$ (Fig.1a) was expressed in stable transgenic plants from its native promoter. The CTR1p:GFP- $\triangle N T$-gCTR1 transgene did not rescue ctr1-2 in etiolated and light-grown plants (Fig. 2a and Supplementary Fig.1). Consistent with this, CTR1p:GFP- $\triangle N T-g C T R 1$ seedlings showed constitutive expression of EBF1 that was comparable to that in $c t r 1-2$ (Fig.2b). The failure of $\Delta N T$-CTR1 transgene to complement $\operatorname{ctr} 1-2$ could result from the reduced ER membrane targeting of $\triangle \mathrm{NT}$-CTR1 protein. $\triangle \mathrm{NT}$-CTR1 was indeed constitutively localized to the nucleus in both dark- or light-grown seedlings, whether expressed from its own or the CaMV 35S promoter (Fig.2c and 2d).

We explored whether the binding of CTR1 to the ethylene receptors might play a role in its nuclear localization. The ctr1-8 mutation blocks the interaction of CTR1 with ETR1 but does not affect intrinsic kinase activity (Fig.1a) ${ }^{25}$. Consistent with the weak hypermorphic nature of ctr1-8, the GFP-CTR $1^{\text {ctr1-8 }}$ transgene partially complemented $c t r 1-2$ in both light- and dark-grown seedlings (Fig.2e and Supplementary Fig.1). Previous fractionation studies demonstrated that ctr1-8 mutant protein was mainly found in a soluble fraction, with a minor population still associated with a membrane fraction ${ }^{15}$, in contrast to the predominant ER localization of wildtype (WT) CTR1. Confirming those prior results, we still observed that GFP-CTR1 $1^{\text {ctr1-8 }}$ localized to the ER (Fig.2f), which likely underlies the partial rescue of ctr1-2 (Fig.2E and Supplementary Fig.1). However, surprisingly, GFP-CTR $1^{\mathrm{ctr1}-8}$ did not translocate to the nucleus in either etiolated- or light-grown seedlings, regardless of ACC treatment (Fig.2g and $2 h$ ). This suggests that interaction with the ethylene receptors is required for nuclear translocation of full-length CTR1, and that modification of CTR1 in response to ethylene at the ER may relieve inhibition of nuclear localization by the $\mathrm{N}$-terminal domain.

\section{The nuclear movement of CTR1 is independent of kinase activity.}


To address the role of kinase activity in CTR1 localization, we expressed a catalytically inactive ctr1 mutant (CTR1p:GFP-gCTR $\left.1^{c t r 1-1}\right)$ in the ctr1-2 mutant background by introducing ctr1-1 (D694E) mutation (Fig.1a) ${ }^{25}$. The $C T R 1^{\text {ctr1-1 }}$ transgene failed to rescue $c t r 1-2$ in either the light or the dark (Fig.3a, 3b, and Supplementary Fig.1), consistent with its hypomorphic nature. Similar to the WT CTR1, full-length GFP-CTR $1^{\text {ctr1-1 }}$ expressed from its native promoter translocated to the nucleus upon ACC treatment (Fig.3b). However, under the strong CaMV 35S promoter, GFP-CTR $1^{\text {ctr1-1 }}$ constitutively localized in the nucleus, like WT CTR1 (Fig.3d). Similar to WT $\triangle \mathrm{NT}$-CTR1 protein, inactive $\Delta \mathrm{NT}$-CTR $1^{\text {ctr1-1 }}$ expressed from either the CaMV $35 \mathrm{~S}$ or

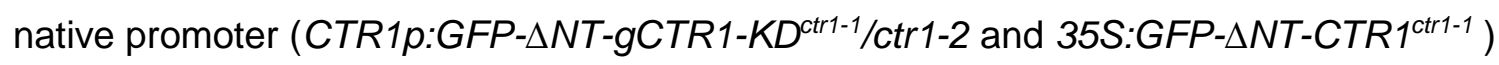

10 constitutively localized to the nucleus in dark and light conditions (Fig.3e and 3f). The $\Delta N T$ $C T R^{\text {ctr1-1 }}$ transgene did not rescue ctr1-2 in either dark- or light-grown seedlings (Supplementary Fig.1 and Fig.2).

$\Delta$ NT-CTR1 autophosphorylates on four residues (S703/T704/S707/S710) located within the activation loop, and this autophosphorylation is critical for CTR1 kinase activity and homodimer formation ${ }^{26}$. To test the role of this autophosphorylation, we altered three residues (T704/S707/S710) to Ala (CTR1 $\left.{ }^{\mathrm{AAA}}\right)$, which has been shown to disrupt homodimer formation ${ }^{26}$. We confirmed that $\triangle \mathrm{NT}-\mathrm{CTR} 1^{\mathrm{AAA}}$ is catalytically inactive using EIN2 as substrate; WT $\Delta \mathrm{NT}$ CTR1, but not $\triangle$ NT-CTR1 ${ }^{A A A}$, phosphorylated EIN2-CEND when co-expressed in Arabidopsis mesophyll protoplasts (Fig.3g). $\Delta$ NT-CTR1 ${ }^{\text {AAA }}$ also failed to form homodimer unlike WT $\Delta$ NTCTR1 in the yeast-2-hybrid assay (Fig.3h). Similar to WT $\triangle$ NT-CTR1, $\triangle$ NT-CTR1-KDAAA was constitutively localized to the nucleus (Fig.3i). Together, these results indicate that kinase activity and homodimerization are not required for CTR1 nuclear translocation.

\section{CTR1 mediates fast growth recovery of seedlings following ethylene-induced growth}


Both ctr1-1 and ctr1-8 mutants show constitutive ethylene responses ${ }^{25}$ despite the observation that $\mathrm{CTR} 1^{\mathrm{ctr} 1-1}$ and $\mathrm{CTR} 1^{\mathrm{ctr} 1-8}$ proteins respond differentially to ethylene for nuclear translocation (Fig.2f, 2g, 2h, 3c, and 3d). This indicates that CTR1 nuclear movement does not control the primary ethylene response, but rather may influence ethylene response kinetics by fine-tuning nuclear ethylene signaling. To test this hypothesis, we measured ethylene growth response kinetics of hypocotyls of etiolated seedlings, which has been widely exploited to analyze ethylene mutants ${ }^{21,27}$. There are two phases of growth inhibition of WT Arabidopsis hypocotyls in response to ethylene; phase I begins 10 min after ethylene treatment and is characterized by a rapid deceleration in growth rate. After a transient (15 min) plateau in growth rate, phase II growth inhibition is initiated with further suppression of growth, lasting 30 min until the growth rate reaches a new, low steady-state rate ${ }^{21,27}$. Genetic studies have revealed that EIN2 is necessary for both phases, but only phase II requires EIN3/EIL1 ${ }^{28}$. Interestingly, upon removal of ethylene during phase II, hypocotyl growth rapidly recovers to the pre-treatment growth rate within $90 \min ^{21,27}$, which indicates the existence of a mechanism to rapidly shut off the ethylene response.

To examine if nuclear-localized CTR1 plays a role in the rapid growth inhibition when ethylene is added or in recovery kinetics when ethylene is removed, we performed time-lapse analysis of the ethylene response growth kinetics of wild type and ctr1-8 mutant seedlings (Fig. 4). Upon ethylene exposure, ctr1-8 seedlings had similar onset and strength of phase I growth inhibition as compared to WT seedlings, demonstrating the normal ethylene response of the ctr1-8. However, the ctr1-8 mutant recovered approximately 90 min later than the WT after removal of ethylene. The delayed hypocotyl growth recovery in ctr1-8 resembled that reported in previous studies with EIN3 overexpression or loss of EBF2 ${ }^{22}$, suggesting that nuclear-localized CTR1 could removes excess EIN3/EIL via EBF2, thus leading to the fast growth recovery. Interestingly, 35S:GFP-CTR1 $1^{\text {ctr1-8 }}$ hypocotyls showed growth recovery kinetics nearly identical to 
those of the WT (Fig.4), presumably due to the existing endogenous CTR1 in 35S:GFPCTR $1^{\text {ctr1-8 }}$ seedlings.

Next, we examined the recovery kinetics of seedlings overexpressing GFP- $\Delta$ NT-CTR1 or GFP- $\triangle \mathrm{NT}$-CTR $1^{\text {ctr1-1 }}$, both of which showed strong constitutive nuclear localization and exhibited an ethylene response in the dark (Fig. 2d and 3f). Despite the constitutive nuclear localization of GFP- $\Delta$ NT-CTR 1 or GFP- $\Delta$ NT-CTR $1^{\text {ctr1-1 }}$, both seedlings only showed slightly faster recovery kinetics (Supplementary Fig. 3). This might be attributed to that the levels of nuclear-localized endogenous CTR1 reaching to the threshold levels that lead to the rapid growth recovery. Together, these results suggest that the translocation of CTR1 to the nucleus

10 plays a role in fast-growth recovery upon removal of ethylene.

\section{CTR1 promotes degradation of EIN3 via EBFs without kinase activity.}

The correlation between nuclear-localized CTR1 and the fast growth recovery kinetics suggests that CTR1 down-regulates nuclear ethylene responses. Thus, we explored if CTR1 modulates EIN3 function in the nucleus in an ethylene-dependent manner. In transiently transformed Arabidopsis protoplasts, the EIN3-dependent activation of a reporter (EBS-LUC), which contains four copies of the EIN3-binding sequence (EBS) fused with the luciferase gene (LUC), was approximately $40 \%$ lower when CTR1 was co-expressed with EIN3 in the presence of ACC (Fig.5a and 5b). The co-expression of $\Delta \mathrm{NT}-\mathrm{CTR} 1^{\mathrm{ctr1}-1}$ with EIN3 also resulted in a reduction in EIN3-mediated EBS-LUC activation, but this effect was independent of exogenous ACC

(Fig.5c). To further examine the underlying mechanism, we investigated CTR1 interactions with EIN3 and with other ethylene signaling components in the nucleus using yeast-2-hybrid and BiFC assays. Full-length CTR1 autoactivates in the yeast-two-hybrid assay, and thus CTR1 kinase domain was employed for this analysis (Supplementary Fig.4). Both $\triangle$ NT-CTR1 and $\triangle \mathrm{NT}-\mathrm{CTR} 1^{\mathrm{ctr1}-1}$ interacted with EIN2-CEND, EBF1, and EBF2, but not with EIN3 (Fig.5d). Fulllength CTR1 also interacted with the EBFs in a BiFC assay (Fig.5e). EIN2-CEND reconstituted 
YFP signals with its known nuclear interacting protein, EIN2 NUCLEAR-ASSOCIATED PROTEIN 1 (ENAP I) in the nucleus (Supplementary Fig.5) ${ }^{29}$, showing that EIN2-CEND was present in the nucleus. Full-length CTR1 interacted with EBF1 and 2 regardless of ACC treatment (Fig.5e and Supplementary Fig.6), which was consistent with constitutive nuclear localization of CTR1 in CaMV 35 S overexpression lines (Fig.1h). Nuclear-localized $\Delta$ NTCTR $1^{\text {ctr1-1 }}$ reduced EIN3 protein levels, but not the abundance of EIN3 transcripts (Fig.5f and $5 \mathrm{~g})$. Assays of protein stability revealed that $\Delta \mathrm{NT}-\mathrm{CTR} 1^{\mathrm{ctr} 1-1}$ substantially shortened the half-life of EIN3 (Fig.5h). However, $\Delta$ NT-CTR1 ${ }^{\text {crr1-1 }}$ did not affect EIN3 stability in the absence of EBF2 (Fig.5i), demonstrating CTR1 regulates EIN3 protein stability via EBFs. Consistent with an increased turnover of EIN3, seedlings overexpressing GFP- $\Delta$ NT-CTR $1^{\text {ctr1-1 }}$ displayed a longer hypocotyl than WT in the absence of ACC. (Fig.5j). Unlike EIN3, the stability of EIN2-CEND protein was not affected by $\Delta \mathrm{NT}-\mathrm{CTR} 1^{\mathrm{ctr} 1-1}$ (Fig.5k). Importantly, we did not detect any phosphorylation of EBF2 by CTR1 in vitro (Fig.5I), consistent with the observation that CTR1 kinase activity is not involved in the regulation of EIN3 protein stability. Together these results demonstrate that upon translocation to the nucleus, a direct interaction between CTR1 and EBFs which is independent of CTR1 kinase activity stimulates EBF-mediated degradation of EIN3 (Fig.7).

\section{The increased nuclear accumulation of CTR1 enhances stress tolerance to abiotic stress.}

Timely recovery after stress removal is a critical factor for plant's resilience. Both seedlings overexpressing GFP- $\Delta$ NT-CTR1 or GFP- $\Delta$ NT-CTR $1^{\text {ctr1-1 }}$ demonstrated the dampening of ethylene signaling as well as fast growth recovery after ethylene removal in the dark (Fig.4). Additionally, the large fraction of both $\Delta \mathrm{NT}$-CTR1 and $\Delta \mathrm{NT}-\mathrm{CTR} 1^{\mathrm{ctr} 1-1}$ constitutively localized in the nucleus in both dark- and light-grown seedlings (Fig.3c, 3d, 4e, and 4f). Therefore, we asked whether 35S:GFP- $\triangle N T-C T R 1^{\text {ctr1-1 }}$ plants have enhanced stress tolerance. Since nuclearlocalized CTR1 is likely an inactive form, we used 35S:GFP- $\triangle N T$-CTR $1^{\text {ctr1-1 }}$ plants for 
investigating the effect of increased nuclear-localized CTR1 in plant's stress response. To determine the effect of water deficit stress, one-week-old GFP- $\triangle N T-C T R 1^{1 t r 1-1}$ seedlings were transferred to MS medium with $20 \%$ PEG to examine the effect of dehydration. Compared to WT, 35S:GFP- $\triangle N T-C T R 1^{\text {ctr1-1 }}$ seedlings displayed only slight growth inhibition and expressed significantly higher levels of drought-responsive genes. (Fig.6a, 6b, and Supplementary Fig.7). Furthermore, we grew 2-week-old WT and GFP- $N$ NT-CTR $1^{\text {ctr1-1 }}$ seedlings on soil without water for 22 days to observe the long-term effect of water stress. Both WT and 35S:GFP- $\triangle N T$ $C T R 1^{\text {ctr1-1 }}$ plants displayed similar drought stress symptoms (Fig.6c). However, 35S:GFP- $\triangle N T$ $C T R 1^{\text {ctr1-1 }}$ plants looked bigger and more turgid compared to WT after the stress. After 7-days of re-watering, the majority of $35 S: G F P-\triangle N T-C T R 1^{\text {ctr1-1 }}$ plants recovered from the stress, whereas the WT plants did not (Fig.6c). Similarly, 35S:GFP- $\triangle N T$-CTR $1^{\text {ctr1-1 }}$ seedlings showed a higher survival rate $(\sim 45 \%)$ than WT $(\sim 12 \%)$ on medium with $175 \mathrm{mM} \mathrm{NaCl}$ and had higher expression of salt-responsive genes upon salt stress (Fig.6d, 6e, 6f and Supplementary Fig.7). Consistent with the plate-salt stress results, 35S:GFP- $\triangle N T-C T R 1^{c t r 1-1}$ plants showed strong salt-tolerance phenotypes on soil irrigated with $300 \mathrm{mM} \mathrm{NaCl}$ for 4-weeks (Fig.6g). These results demonstrate that an increasing amount of nuclear-localized CTR1 confers enhanced tolerance to abiotic stress, likely through reinforcing the fine-tuning of ethylene signaling during the stress acclimation and via CTR1-induced rapid suppression of ethylene response after stress removal.

Upon perception of environmental signals, the specificity of cellular responses to the stress are mediated by the spatial and temporal dynamics of downstream signaling networks. Here we provide convincing evidence for the identification of a mechanism linking the spatiotemporal regulation of CTR1 to organismal stress responses. We discover that upon inactivation by ethylene, CTR1 re-locates from the ER to the nucleus where CTR1 downregulates the nuclear ethylene responses by downregulating EIN3 via activation of EBF's function. Remarkably, we 
further discovered that the nuclear movement of CTR1 is tightly associated with a plant's resilience to abiotic stress. The stress tolerance traits conferred by the nuclear-localized CTR1 are likely due to that nuclear-localized CTR1 mitigates the harmful effect of ethylene signaling during stress acclimation and also promote timely growth recovery upon the stress removal. These CTR1-induced stress tolerance traits present a potential key to manipulating plant's adaptability to abiotic stresses such as drought and salinity.

Our results suggest that there is a tight regulation on equilibrium between EIN2mediated EIN3 activation and CTR1-mediated EIN3 inhibition in the nucleus, which is critical for optimal ethylene response in various stress conditions. Nuclear accumulation of CTR1 was first 10 detectable approximately 30 min after ethylene treatment in our studies (Fig.1j), which is coincident with the initiation time of the first plateau after the phase I inhibition and is slower than accumulation of EIN2 ${ }^{17}$. These results indicate that nuclear-localized CTR1 may hamper EIN2-induced activation of EIN3 to initiate ethylene responses during this plateau. However, due to the continued binding of ethylene to the receptors, the EIN2-induced activation of EIN3 would override the effects of CTR1-mediated downregulation of EIN3 in the presence of ethylene, thus promoting continued growth suppression. Upon removing ethylene gas, the equilibrium may rapidly shift from EIN2-mediated activation of EIN3 to CTR1-mediated EIN3 degradation via an unknown mechanism, initiating rapid growth recovery.

The ctr1-8 mutant seedlings still showed some levels of growth recovery despite its complete failure to translocate to the nucleus in response to ethylene (Fig.4). These results imply the existence of other recovery mechanisms that are not dependent on nuclear CTR1 for controlling the fast growth recovery, such as ethylene-receptor-mediated inactivation of ethylene responses ${ }^{28}$.

The failure of CTR $1^{\text {ctr1-8 }}$ protein to translocate to the nucleus was surprising as CTR $1^{\text {ctr1-8 }}$ only weakly interacted with the ETR1 receptor, suggesting the majority of CTR $1^{\operatorname{ctr} 1-8}$ was cytoplasmic as indicated by previous studies. This result implies that CTR1 recrement to the ER 
and its interaction with ethylene receptors are necessary for ethylene-induced CTR1 nuclear targeting. Based on the constitutive nuclear localization of CTR1-KD without the N-terminus, we speculate that $\operatorname{ctr} 1-8$ mutation inhibits conformational changes of the CTR $1^{\text {trr1-8 }}$ protein, which relieves the $\mathrm{N}$-terminus-mediated inhibition on CTR1 nuclear translocation. It is also possible that $c$ tr1-8 mutation prevents the mutant CTR1 protein from interacting with unknown cargo protein that brings CTR 1 to the nucleus as CTR1 does not have canonical nuclear localization sequences.

Due to the absence of canonical nuclear localization sequences in CTR1, how it translocates from the ER to the nucleus and whether other ethylene-activated components are

10 involved in this process remains to be determined. Furthermore, identification of CTR1independent alternative pathway that controls the rapid growth recovery of seedling will bring more insights into the mechanistic understanding of ethylene response regulation. Ethylene interacts with a myriad of internal and external stimuli, regulating plant growth and stress responses. Thus, it will be of great interest to revisit crosstalk with other pathways, taking into consideration the nuclear role of CTR1.

\section{References and Notes:}

Acknowledgments: We thank Alison Delong for critical reading of the manuscript and Anna Stepanova for providing EBS-GUS-pCAMBIA-1381Z construct and Purdue Imaging Facility for confocal microscope service. This work was supported by grants from NSF (MCB- 1817286) and Ralph W. and Grace M Showalter Research Trust to GMY, NSF to JJK (IOS-1856431) and B.M.B (MCB-1817304).

Author contributions: GMY and JJK conceived the experiments; GMY planned and supervised experiments; HYL and DHS conducted experiments except ethylene-response kinetics and BiFC; $\mathrm{AB}$ and $\mathrm{BMB}$ conducted ethylene response kinetics analysis; HLP performed 
BiFC and physiology experiments. CP performed BiFC; GMY, HYL, DHS, JJK, BMB, and HLP contributed to the interpretation of the data; GMY wrote the manuscript with support from JJK and BMB. HYL and DHS equally contributed to the manuscript.

Declaration of Interests: The authors declare no competing interests.

Data and materials availability: All data is available in the main text or the supplementary materials.

\section{Materials and Methods:}

\section{Plant materials and growth conditions}

Arabidopsis Thaliana Col-0 was used as the wild-type (WT) reference throughout the study. All plants were grown in either long-day or short-day conditions at $22^{\circ} \mathrm{C} \pm 2^{\circ} \mathrm{C}$ or in vitro on Murashige and Skoog (MS) Basal Medium supplemented with 0.8\% plant agar (pH 5.7) in a continuous light chamber at $21^{\circ} \mathrm{C}$. All plants used were homozygous or T2 lines. Homozygous transgenic lines were identified by the segregation of antibiotic resistance followed by confirmation of protein expression via immunoblot analysis.

\section{CTR1 Constructs and Site-Directed in Vitro Mutagenesis for Arabidopsis Transformation.}

All molecular cloning was performed using the Gateway (Invitrogen) or infusion cloning (Takara Bio USA) strategies otherwise specified. Table. S1, S2, and S3 list all primers used for cloning and mutagenesis. To create CTR1p-GFP-gCTR1 construct, we PCR-amplified three separate overlapping fragments (CTR1 promoter, YFP, and the genomic fragment of CTR1). $0.96 \mathrm{~Kb}$ of CTR1 promoter and $4.7 \mathrm{~kb}$ of full length CTR1 genomic fragment were amplified using Col-0 genomic DNA as a template and the YFP coding sequences were amplified using binary vector pEarleyGate 104. Three overlapping fragments were subsequently subjected to infusion reaction with Stul- and Xbal-digested pEarleyGate 104, creating the GFP-full-length CTR1 clone 
in pEarleyGate 104 backbone. Mutations on the genomic CTR1 fragment (G354E ctr1-8 or D694E ctr1-1 mutation) were introduced into the WT genomic fragment via PCR-based mutagenesis and the resulting fragments were used for infusion reactions as described above. CTR1p-YFP-gCTR1 and its mutant variant constructs were also constructed identically as above except using YFP instead of GFP. To construct 35S:GFP-CTR1, GFP-CTR1 ${ }^{\text {ctr1-1 }}$, GFP$\triangle N T-C T R 1, G F P-\triangle N T-C T R 1^{c t r 1-1}$, and GFP-CTR1 $1^{\text {ctr1-8 }}$, the coding sequences of full length or kinase domain of CTR1 were cloned into pENTR entry vector and were subsequently transferred to binary vector pSITE2CA. Mutations were introduced in the coding sequences of CTR1 in the pENTR vector and the sequences were further transferred to pSITE2CA.

\section{Bimolecular Fluorescence Complementation Constructs}

The following coding sequences including a stop codon were transferred from pENTR vector into pCL112 to generate N-terminal nYFP-fusions: CTR1, CTR $1^{\text {ctr1-1 }}, C T R 1^{\text {ctr1-8 }}, \Delta N T-C T R 1^{\text {ctr1-1. }}$. The following coding sequences without a stop codon were transferred from pENTR vector into pBAT-YFPc to generate C-terminal cYFP-fusion: ETR1, EIN3, EIN2-CEND, and CIP8. The coding sequences of EBF1 and EBF2 in pENTR were transferred into pCL113, creating cYFPEBF2.

\section{Transactivation assay}

To construct effector plasmids, the coding sequences of EIN3, CTR $1^{\text {ctr1-1 }}$, or $\triangle N T-C T R 1^{\text {ctr1-1 }}$ in pENTR gateway vector were transferred into pEarleyGate 203, creating a myc-tagged fusion protein. To generate a reporter plasmid, we PCR-amplified 4XEBS with minimal 35S promoter using EBS-GUS-pCAMBIA-1381Z as a template. The coding sequences of Luciferase was PCR-amplified using pEGB 35S:Luciferase:Tnos (Addgene, GB0110). The amplified two fragments and Stul- and Xbal-digested pEarleyGate 104 vector were further subjected to 
infusion reaction to create a $4 x E B S: \min 35 S p$-Luciferase. All final plasmid constructs were verified by DNA sequencing. We used pEGB 35S:Renilla:Tnos (Addgene, GB0109) as an internal control plasmid. To perform transactivation assay, Arabidopsis mesophyll protoplasts were isolated from 3-week-old Arabidopsis grown under a $12 \mathrm{~h}$ light/12 $\mathrm{h}$ dark regime at $22^{\circ} \mathrm{C}$ as previously described. For transfection, $5 \times 10^{5}$ protoplasts were incubated with the appropriate effector $(5 \mu \mathrm{g})$ and reporter plasmid DNA $(2.5 \mu \mathrm{g})$ in $20 \%$ polyethylene glycol 4000 (Sigma-Aldrich) for $5 \mathrm{~min}$. Transfected protoplasts were then washed twice with W5 solution and incubated for $12-16 \mathrm{~h}$ followed by lysis with Cell Culture Lysis Solution (Promega). The activities of LUC and rLUC in cell lysates were measured using a Dual-Luciferase Assay System (Promega).

\section{Phostag gel analysis}

Preparation of Phos-Tag polyacrylamide gels and subsequent immunoblotting was performed according to the manufacturer's instructions. Phos-Tag gel was prepared using $5 \mathrm{ml}$ of $8 \%$ acrylamide and $20 \mu \mathrm{M}$ Phos-tag (Wako) for the resolving gel and $4 \mathrm{~mL}$ of $4.5 \%$ acrylamide for the stacking gel. After completion of electorphoresis, the gel was incubated in a $100 \mathrm{~mL}$ transfer buffer containing $0.2 \%(w / v)$ SDS for $30 \mathrm{~min}$. Protein was blotted onto Nitrocellulose followed by blocking with $5 \%$ nonfat milk. The membranes were then probed with 1:5000 dilution of Roche Anti-GFP (SigmaAldrich Cat\#. 11814460001) or 1:5000 dilution of anti-mouse HRP secondary antibody.

\section{Time-lapse growth recovery analysis}

To measure ethylene response growth kinetics of seedlings, seedlings were grown on vertically oriented petri plates in darkness to a height of 3 to $4 \mathrm{~mm}(42-46 \mathrm{~h})$ before the beginning of growth-rate measurements. The agar plates were placed vertically in a holder and fitted with a 
lid for continuous gas flow $\left(100 \mathrm{~mL} \mathrm{~min}^{-1}\right)$. Seedlings were grown in air for $1 \mathrm{~h}$ followed by application of ethylene (typically 1 or $10 \mathrm{ppm}$ ) for $2 \mathrm{~h}$, followed by removal of ethylene. Images were acquired every 5 min using a CCD camera fitted with a close-focus lens with illumination provided by infrared LEDs. The growth rate of the hypocotyls in every time interval was then calculated. Under these conditions, the equilibration time of the chamber at these flow rates was approximately $30 \mathrm{sec}$, which was much faster than the image acquisition time. From this, we determined how various mutations affect growth inhibition kinetics when ethylene was added and recovery kinetics when it was removed.

\section{Yeast-2-hybrid}

The coding sequences of the full-length CTR1 or kinase domain of CTR1 ( $\triangle N T$-CTR1) with or without ctr1-1 mutation (D694E) in pENTR GW entry vector were transferred into pGBDT7 or pGADT7. The resulting bait clones were paired with EIN2-CEND, EIN3, EBF1, or EBF2 in pGBDT7 or pGADT7 vector and tested their interaction in yeast. Positive interactions between prey and bait were selected on medium lacked histidine, tryptophan, leucine, and adenine. Due to autoactivation by full-length CTR1 in pGBDT7, $\triangle N T$-CTR1 or $\triangle N T$-CTR1 $1^{\text {ctr } 1-1}$ was used to generate CTR1 bait constructs.

\section{Confocal microscopy}

All imaging of GFP, YFP, and mCherry were carried out using a laser-scanning confocal microscope (Zeiss LSM880 upright). Samples were directly mounted on a glass slide in water. For imaging of Arabidopsis seedlings transformed with CTR1 constructs, seedlings were grown on MS media supplemented with or without $10 \mu \mathrm{M} \mathrm{AgNO}_{3}$ in the dark for 3 days (3-d). For ACC treatment, seedlings on $\mathrm{MS}$ without $\mathrm{AgNO}_{3}$ were treated with $200 \mu \mathrm{M} \mathrm{ACC}$ dissolved in water for $2 \mathrm{~h}$. For ethylene treatment, seedlings were grown directly on MS media in GC vials for 3 days. The GC vials subsequently were capped and injected with 1 ppm ethylene gas and 
incubated for 2 h. For nuclear imaging, 3-d-old dark-grown seedlings were treated with $200 \mu \mathrm{M}$ ACC for $2 \mathrm{~h}$, followed by incubation with Hoechst33342 solution (Invitrogen) for 30 min and brief washing before mounting on slides. For imaging light-grown seedlings, plants were grown on MS in constant light for 5 days. For imaging of fluorescence signals in protoplasts, transfected protoplasts were incubated with $200 \mu \mathrm{M}$ ACC for $2 \mathrm{~h}$ in dark and subjected to examination. All imaging was performed using more than 3 independent biological replicates of at least 3 independent lines. To image BiFC, leaf disks of infiltrated tobacco leaves with CTR1 and counterpart constructs (EIN2-CEND, EIN3, EBF1, EBF2, ENAP1, and CIP8) were mounted in water on glass slides.

\section{Arabidopsis drought and salt stress experiments.}

For drought recovery experiments, Arabidopsis seedlings were grown on soil in short-day conditions for 14-d and water was withdrawn for 22-d followed by re-watering for 7-d.

For the salt stress treatment, Arabidopsis seedlings were grown on soil in short-day conditions for 14 days. Starting on day 14, the plants were treated with $300 \mathrm{mM} \mathrm{NaCl}$ every 4-d for 28-d, followed by $7-d$ of recovery. The experiment was repeated three times with similar results.

\section{In vitro kinase assay}

A total of $20 \mathrm{ng}$ purified $\mathrm{His}_{6}-\Delta \mathrm{NT}-\mathrm{CTR} 1$ or $\mathrm{His}_{6}-\Delta \mathrm{NT}-\mathrm{CTR} 1^{\mathrm{ctr} 1-1}$ protein was incubated with 100 ng of $\mathrm{His}_{6}$-EIN2 ${ }^{\mathrm{WT}}$-His 6 , $\mathrm{His}_{6}$-EBF2, or $\mathrm{His}_{6}$-EIN3 in kinase reaction buffer [50 mM Tris (pH 7.5), $10 \mathrm{mM} \mathrm{MgCl}_{2}, 1 \times$ Roche Complete Protease Inhibitor mixture, $\left.1 \mu \mathrm{Ci}\left[\mathrm{\gamma}^{-32} \mathrm{P}\right] \mathrm{ATP}\right]$ for $30 \mathrm{~min}$ at room temperature. After incubation, reactions were terminated by boiling in $6 \times$ Laemmli SDS sample buffer for 3 min. Samples were subjected to SDS/PAGE, dried, and visualized by autoradiography. 


\section{Real-Time Quantitative PCR Analysis.}

Total RNA was prepared using RNeasy Plant Mini Kit (QIAGEN) and reverse transcribed using SuperScript II reverse transcriptase (Invitrogen) according to the manufacturers' instructions. Quantitative RT-PCR was performed using PowerUP ${ }^{T M}$ SYBRGreen Master Mix (Applied Biosystems). Primers used are listed in Table S5. Three biological replicates were analyzed with three technical replicates per sample. The relative expression for candidate genes was normalized to Actin 2.

\section{Analysis of EIN3 half-life turnover}

Protoplasts were transfected with GFP-tagged EIN3 with or without GFP-tagged $\triangle N T$-CTR1 ${ }^{\text {ctr } 1-1}$ plasmid using PEG-mediated transformation. GFP plasmid was co-transfected as a transfection control and the difference in the total amount of plasmid was complemented by adding an empty vector. Transfected protoplasts were incubated for $16 \mathrm{~h}$, followed by incubation with $250 \mu \mathrm{M}$ cycloheximide (Sigma-Aldrich). Protoplasts were then harvested at different time points for immunoblotting analysis. 


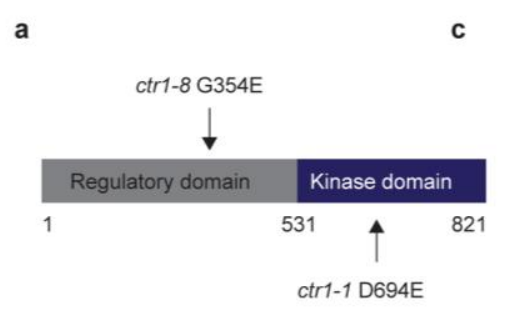

b
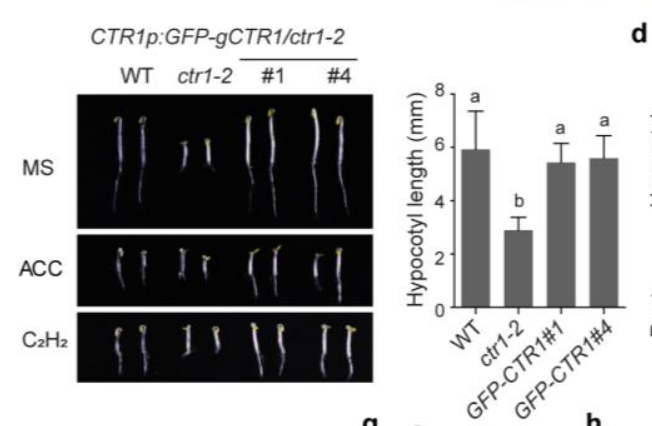

d CTR1p:GFP-gCTR1/ctr1-2

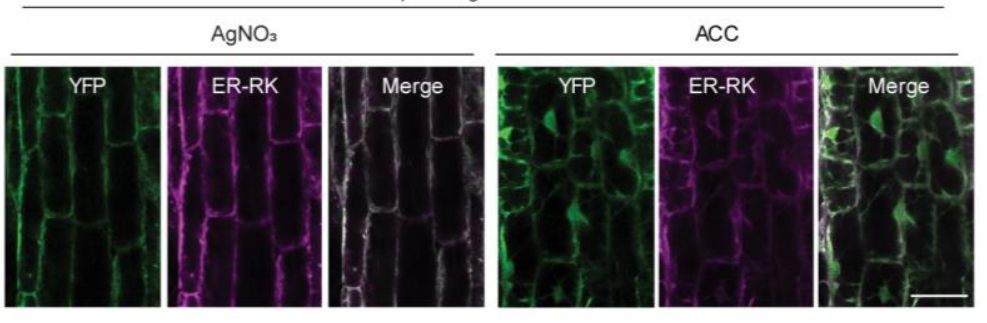

CTR1p:GFP-gCTR1/ctr1-2
GFP Hoechst Merge

e
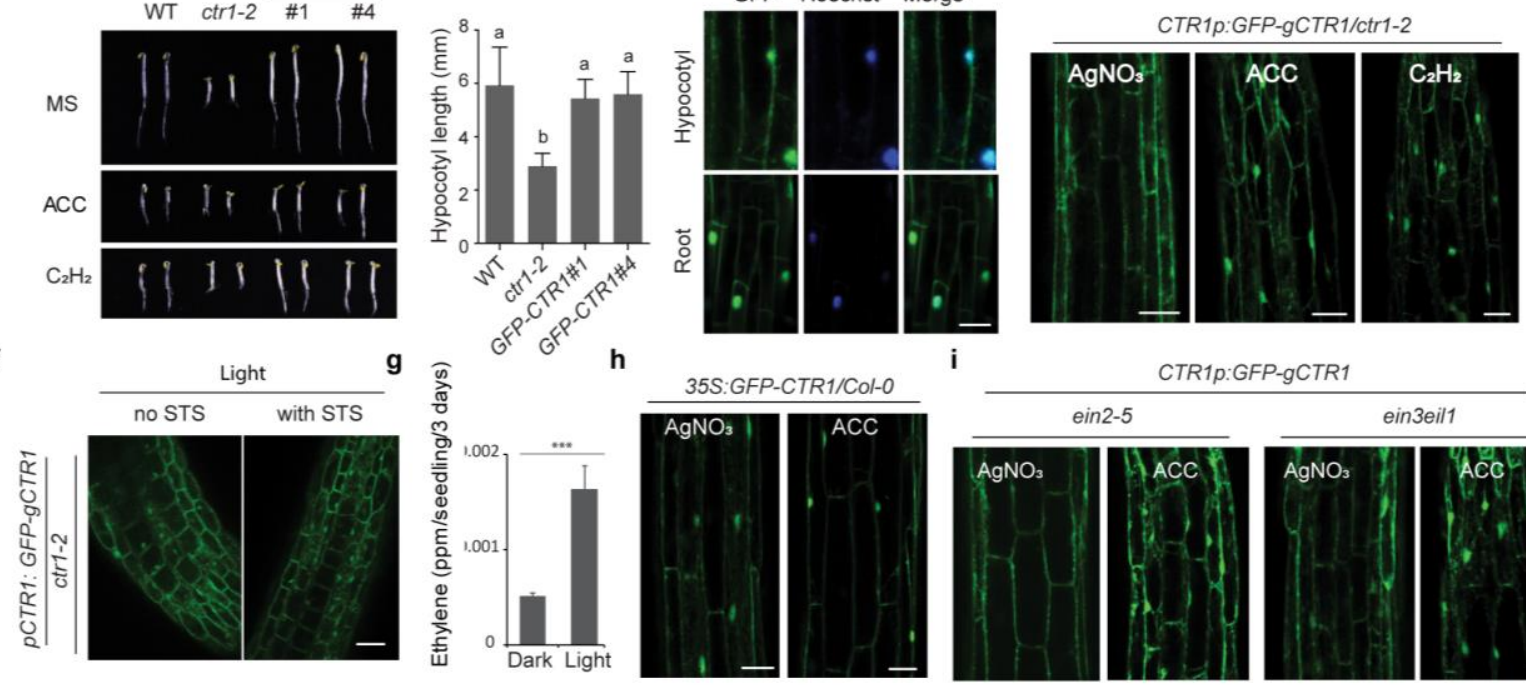

i
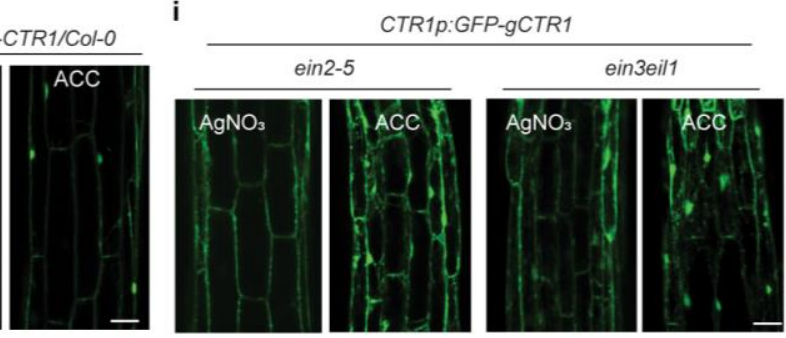

j
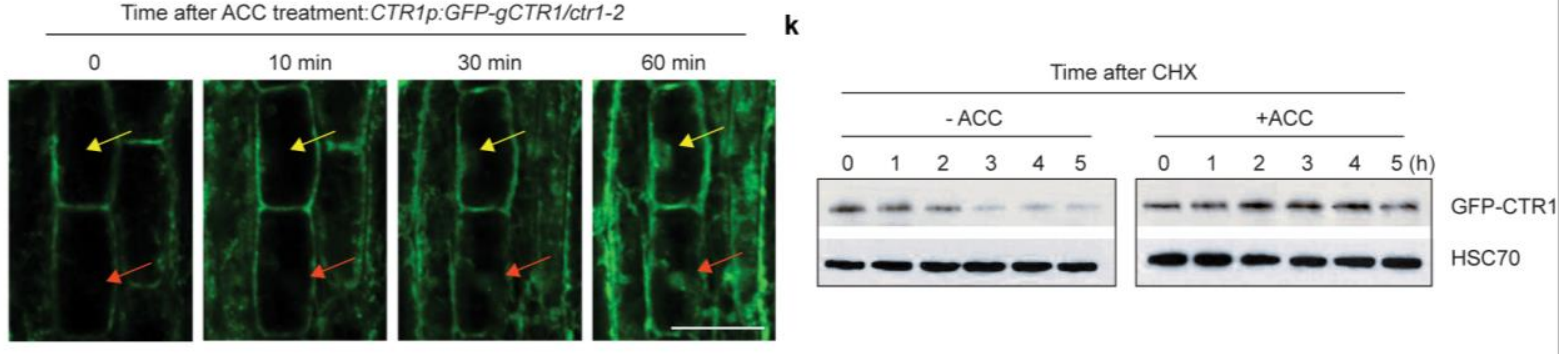

Fig. 1

Fig. 1. Ethylene-activated CTR1 translocation to the nucleus does not require EIN2 and

EIN3. a, Diagram of CTR1 protein domain structure. The position of ctr1 mutant alleles are indicated by arrows. b, The GFP-fused WT genomic CTR1 fragment fully rescues ctr1-2 and confers ethylene response. Seedlings were grown for 3 days (3-d) in the dark with or without

ACC or ethylene. The graph represents the quantification of hypocotyl length of seedlings grown on MS without ACC. MS, Murashige and Skoog medium. Data represent the means and SD (n > 35 biological replicates). c, Three-d-old etiolated seedlings co-expressing GFP-CTR1 and ERRK were grown on $\mathrm{MS}$ medium with $\mathrm{AgNO}_{3}$ or treated with $\mathrm{ACC}$ for $2 \mathrm{~h}$. ER-RK ${ }^{30}$, an mCherry- 
fused ER marker. d, GFP-CTR1 fluorescence overlaps with Hoechst nuclear staining under ACC treatment, showing CTR1 nuclear localization. e, Ethylene and ACC activates CTR1 nuclear translocation. Seedlings were grown on MS medium with or without $10 \mu \mathrm{M} \mathrm{AgNO}_{3}$ in dark. Seedling grown on $\mathrm{MS}$ without $\mathrm{AgNO}_{3}$ were treated with $200 \mu \mathrm{M} \mathrm{ACC}$ for $2 \mathrm{~h}$. For ethylene treatment, seedlings were grown in vials without capping for 3-d, followed by capping and injection of $10 \mathrm{ppm}$ ethylene for $2 \mathrm{~h}$ before imaging. Scale bars, $50 \mu \mathrm{m}$. f, Constitutive nuclear localization of CTR1 in 5-d-old light-grown seedlings. Seedlings expressing GFP-CTR1 from its native promoter in ctr1-2 background was grown on MS medium with or without $50 \mu \mathrm{M}$ silver thiosulfate (STS) in light for 5-d and GFP-CTR1 fluorescence was visualized by confocal

10 microscopy. g, Ethylene biosynthesis in wild-type (WT) Arabidopsis seedlings grown in constant dark or light condition. WT Arabidopsis seedlings were grown in dark or light conditions for 3-d and accumulated total ethylene was measured using gas chromatography. Data represent the means and SD. ${ }^{* *} p<0.001$, Student's $t$-test. $\mathbf{h}$, Overexpression of CTR1 leads to constitutive nuclear localization of CTR1 in dark-grown seedlings. i, Seedlings expressing GFP-CTR1 in ein2-5 or ein3eil1 mutant were grown on $\mathrm{MS}$ medium with or without $\mathrm{AgNO}_{3}$ for 3-d. For $\mathrm{ACC}$ treatment, the seedlings grown on $\mathrm{MS}$ medium without $\mathrm{AgNO}_{3}$ were treated with $\mathrm{ACC}$ for $2 \mathrm{~h}$ before visualization by confocal microscopy. j, Time-lapse image series of hypocotyl cells expressing GFP-CTR1 in 3-d-old etiolated seedlings after exposure to $200 \mu \mathrm{M}$ ACC, visualized by confocal microscopy. Arrows track specific cell nuclei, showing the accumulation of GFP. CTR1 in response to ACC. All scale bars represent $50 \mu \mathrm{m}$, except the scale bar in B. $\mathbf{k}$, The half-life analysis of CTR1. CTR1p:GFP-gCTR1/ctr1-2 seedlings were grown on MS medium with or without $10 \mu \mathrm{M} \mathrm{ACC}$ and the seedlings were subsequently treated with $250 \mu \mathrm{M}$ cycloheximide $(\mathrm{CHX})$ and total protein extracts were used for immunoblotting. 
a

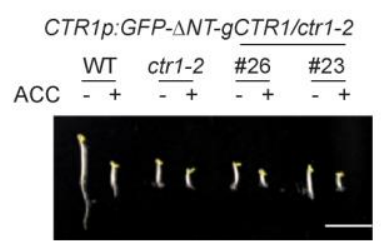

e

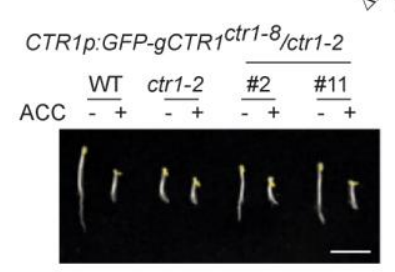

g

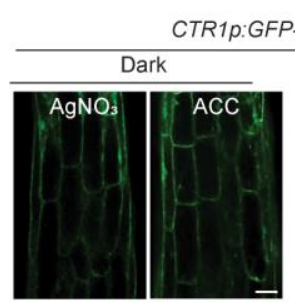

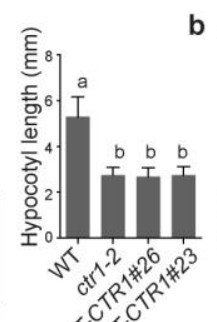

b
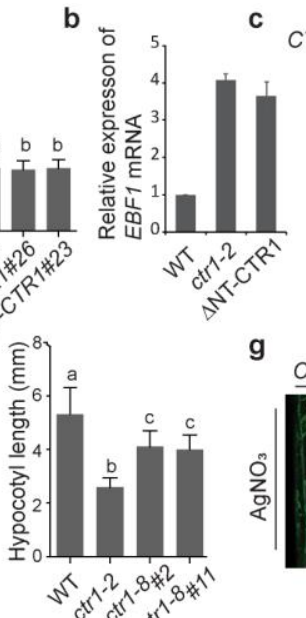

g

CTR1P:YFP-gCTR $1^{\text {Ctr1-8 }} / \mathrm{Col}-0$
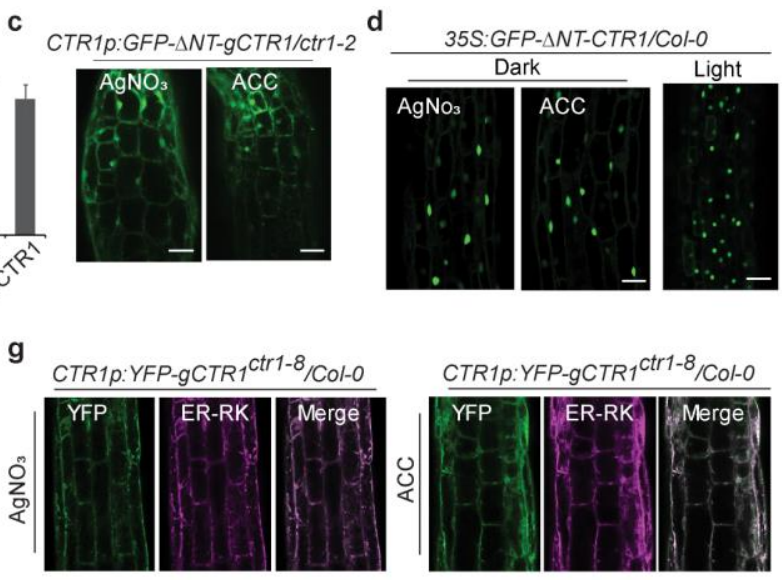

h

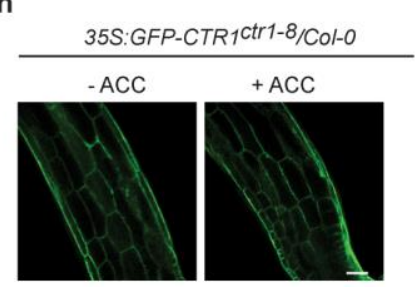

Fig. 2

Fig. 2. The N-terminus of CTR1 inhibits ACC-induced CTR1 nuclear movement. a, Seedlings were grown on MS medium with or without $10 \mu \mathrm{M} \mathrm{ACC} \mathrm{for} \mathrm{3-d} \mathrm{and} \mathrm{photographed.}$ The graph represents the quantification of hypocotyl length of seedlings grown on MS without ACC. Scale bar, $5 \mathrm{~mm}$. Different letters indicate significant differences at $p<0.001$ (One-way ANOVA, post-hoc Tukey's HSD), data represent the means and SD for 3 biological replicates. b, Quantitative gene expression analysis for the ethylene-responsive EBF1 in ctr1-2 and CTR1p: GFP- $\triangle$ NT-gCTR1 seedlings without ACC treatment. Expression was normalized to an Actin control and is presented as relative to the untreated WT control. Data represent the means and SD for three biological replicates. c, Constitutive nuclear localization of GFP- $\triangle N T-C T R 1$ expressed from the native promoter in hypocotyls of dark-grown seedlings. d, Constitutive nuclear localization of GFP- $\triangle$ NT-CTR1 expressed from CaMV 35S promoter in 3-d-old dark or 5-d-old light-grown seedlings. e, Seedlings expressing GFP-CTR1 $1^{\text {ctr1-8 }}$ from the native promoter were grown on MS medium with or without $10 \mu \mathrm{M} \mathrm{ACC}$ for $3-d$ and photographed. The graph 
represents the quantification of hypocotyl length of seedlings grown on MS without ACC. Scale bar, $5 \mathrm{~mm}$. Different letters indicate significant differences at $p<0.001$ (One-way ANOVA, posthoc Tukey's HSD), data represent the means and SD for 3 biological replicates. $\mathbf{f}$, Seedlings coexpressing YFP-CTR $1^{\text {crr1-8 }}$ and ER-RK were grown in the dark and used for the visualization of co-localization. e-h, GFP-CTR $1^{\text {ctr1-8 }}$ does not translocate into the nucleus in both dark and light conditions. Scale bars in C, D, F, G, and H represent $50 \mu \mathrm{m}$. 


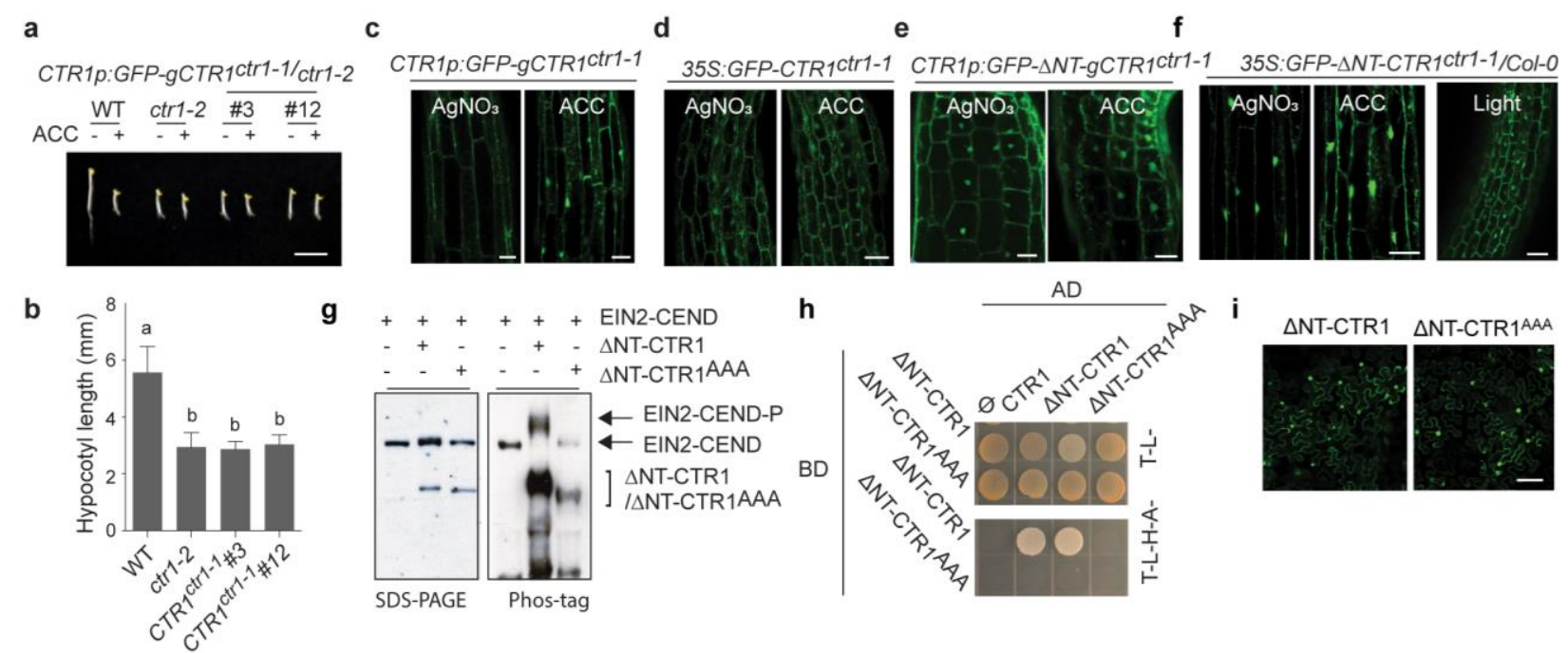

Fig. 3

Fig. 3. The kinase activity of CTR1 is not necessary for ACC-induced CTR1 nuclear translocation. a, Seedlings were grown on MS medium with or without $10 \mu \mathrm{M} \mathrm{ACC}$ for 3-d and photographed. Scale bar, $5 \mathrm{~mm}$. b, Quantification of hypocotyl length of seedlings grown on MS without ACC in (A). Data represent the means and SD. c-d, Seedlings expressing GFPCTR $1^{\text {ctr1-1 }}$ from its native promoter (c) or CaMV 35 S promoter (d) were grown on MS medium with or without $\mathrm{AgNO}_{3}$. For $\mathrm{ACC}$ treatment, the seedlings grown on $\mathrm{MS}$ medium without $\mathrm{AgNO}_{3}$ were further treated with ACC for $2 \mathrm{~h}$ before imaging. e-f, Seedlings expressing GFP- $\triangle \mathrm{NT}$ CTR $1^{\text {ctr1-1 }}$ from its native promoter (e) or CaMV 35 S promoter (f) were grown on MS medium with or without $\mathrm{AgNO}_{3} . \mathbf{g}$, Co-expression of the EIN2-CEND with WT $\triangle$ NT-CTR1 in Arabidopsis protoplasts resulted in a shifted band of EIN2-CEND in a Phos-tag gel, but not in an SDSPAGE, indicating EIN2-CEND phosphorylation by the $\triangle$ NTT-CTR1. Unlike WT $\triangle N T-C T R 1$, coexpression of $\triangle N T$-CTR1 ${ }^{\text {AAA }}$ did not result in a shift of EIN2-CEND band. $\mathbf{h}$, The indicated bate and prey constructs were co-transformed into $\mathrm{AH} 109$ yeast strain and the transformed yeast 15 were grown on selection media. i, Tobacco leaves were infiltrated with agrobacterium transformed with $\Delta \mathrm{NT}$-CTR1 or $\triangle \mathrm{NT}$-CTR1 ${ }^{\mathrm{AAA}}$ plasmid construct, followed by 3-d incubation and 
bioRxiv preprint doi: https://doi.org/10.1101/2020.06.12.148858; this version posted June 13,2020 . The copyright holder for this preprint (which was not certified by peer review) is the author/funder. All rights reserved. No reuse allowed without permission.

visualization of nuclear signals by confocal microscopy. All scale bars represent $50 \mu \mathrm{m}$ except the scale bar in $(\mathbf{a})$. 


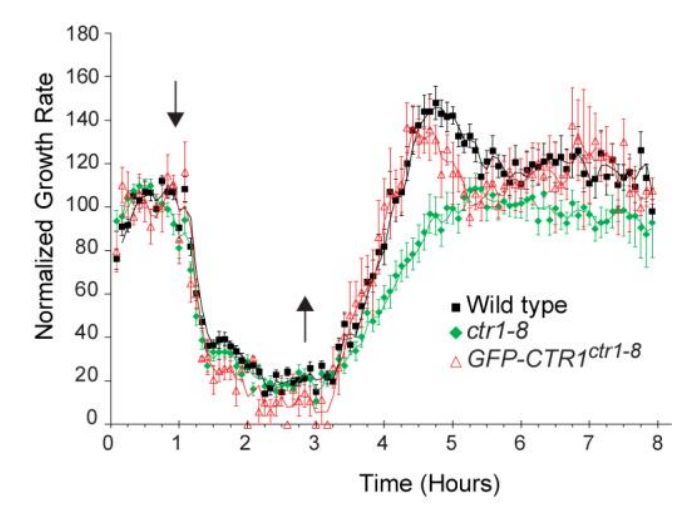

Fig. 4

Fig. 4. The failure of the nuclear translocation of CTR1 inhibits fast growth recovery after ethylene removal. Hypocotyl growth rate in response to ethylene was recorded for $1 \mathrm{~h}$ in air, 10 followed by $2 \mathrm{~h}$ exposure to $10 \mathrm{ppm}$ ethylene, and then $5 \mathrm{~h}$ recovery in air. The ethylene response growth kinetics of WT, ctr1-8, and 35S:GFP-CTR ${ }^{1-8}$ are shown in the graph. Data represent the means and SE. 
a

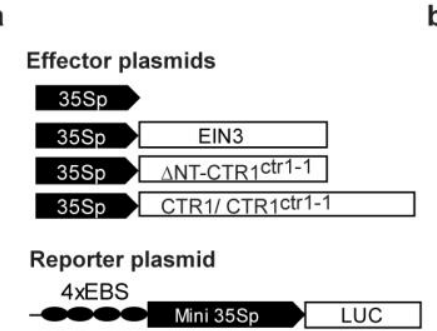

Internal control

$35 \mathrm{Sp} \quad \mathrm{rLUC}$ b

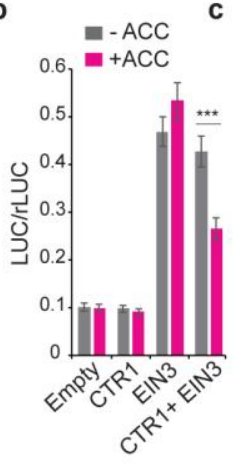

d
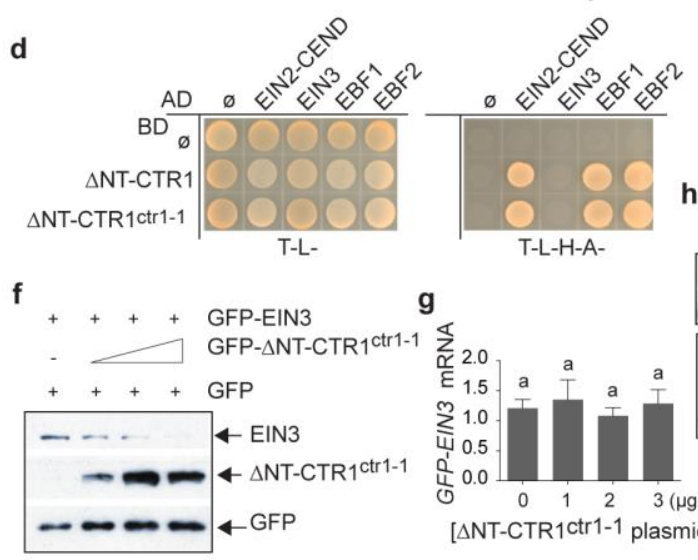

j

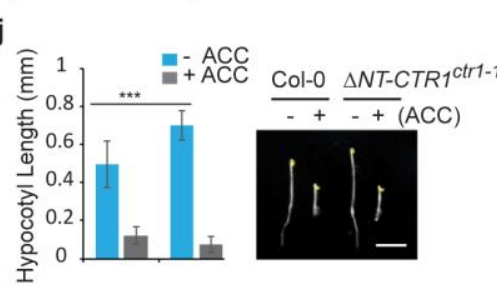

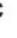

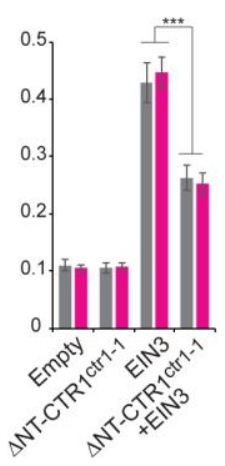

e

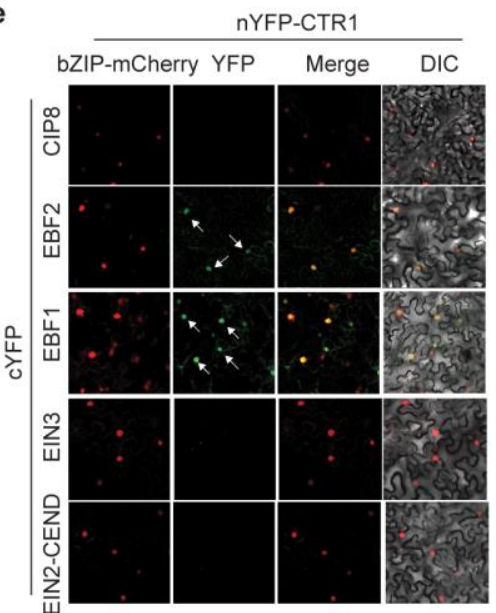

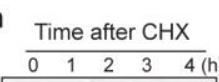
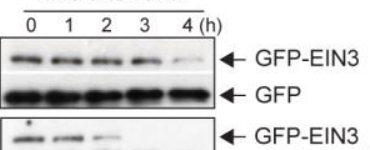

$\div=-\ldots$ GFP-EIN3 4 -

i Time after $\mathrm{CHX}$

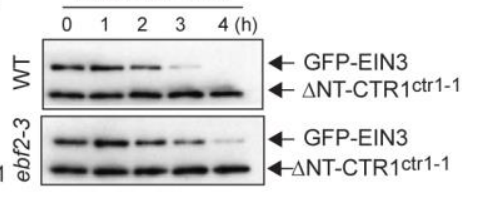

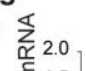
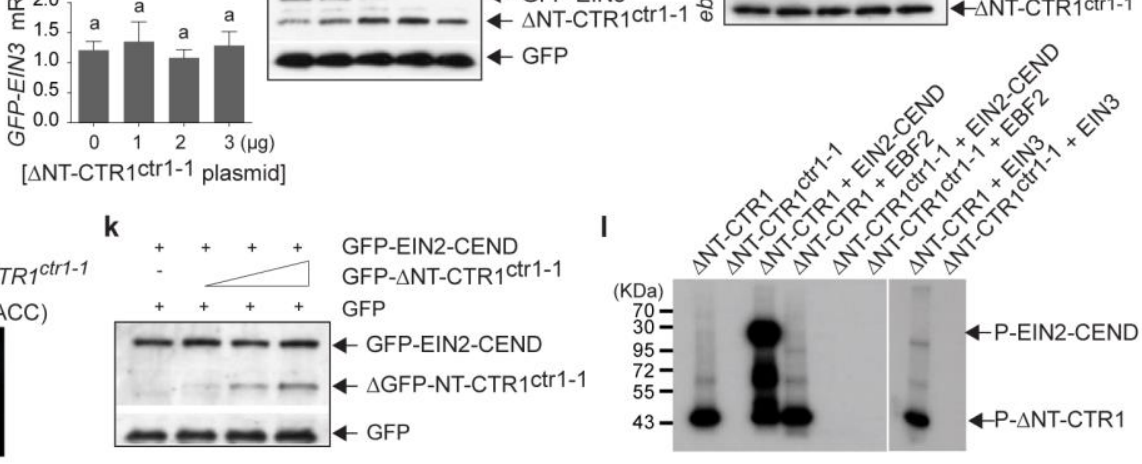

Fig. 5

Fig. 5. Nuclear-localized CTR1 downregulates EIN3 via a non-catalytic function. a,

Schematic diagrams of the effector, reporter, and internal control plasmids used in the transient transactivation assay in Arabidopsis leaf protoplasts. b-c, The effect of CTR1 or $\triangle \mathrm{NT}$-CTR $1^{\text {ctr1-1 }}$ on the EIN3 transactivation of EBS-Luc reporter gene in the presence or absence of ACC. Data represent the means and SD for three biological replicates, ${ }^{* \star *} p<0.001$, Student's $t$-test. $\mathbf{d}$, The indicated bait and prey constructs were co-transformed into AH109 and the growth of the transformed AH109 was monitored on selection media. e, BiFC assay for full-length WT CTR1 and nuclear ethylene signaling proteins in $N$. benthamiana in the presence of ethylene. COP1interacting protein 8 (CIP8) and bZIP-mCherry were used as a negative control and a nuclear subcellular marker, respectively. $\mathbf{f}, \triangle \mathrm{NT}$-CTR $1^{\text {ctr1-1 }}$ promotes the degradation of EIN3. The 
indicated plasmids were co-transfected into Arabidopsis protoplasts and incubated for $16 \mathrm{~h}$, then treated for $2 \mathrm{~h}$ incubation with $200 \mu \mathrm{M}$ ACC. Total protein extracts were prepared and used for immunoblotting using anti-GFP antibody. g, Nuclear-localized CTR1 does not affect the transcript levels of EIN3. Arabidopsis protoplasts were co-transformed with a consistent amount of EIN3 and an increasing amount of $\Delta \mathrm{NT}-\mathrm{CTR} 1^{\mathrm{ctr} 1-1}$ plasmid and incubated for $16 \mathrm{~h}$. Total RNA was extracted from the protoplasts and used for quantitative PCR analysis. Relative gene expression of GFP-EIN3 was normalized to Actin reference gene. Error bars, SD. $p<0.001$ (One-way ANOVA, post-hoc Tukey's HSD), Error bars. SD. h, The effect of $\Delta \mathrm{NT}$-CTR $1^{\text {ctr1-1 }}$ on EIN3 half-life. i, Protoplasts from WT or ebf2-3 mutant plants were isolated and co-transfected with GFP-EIN3 and GFP- $\triangle N T-C T R 1^{\text {ctr1-1 }}$ plasmids and incubated for $16 \mathrm{~h}$. The transfected protoplasts were treated with $\mathrm{CHX}$ and total proteins were extracted after the time indicated. j, Three-d-old dark-grown WT and 35S:GFP- $\triangle N T-C T R 1^{\text {ctr1-1 }}$ seedlings treated with or without 1 $\mu \mathrm{M}$ ACC. Representative seedlings are shown in comparison with WT. Quantification of hypocotyl length of $35 S: G F P-\triangle N T-C T R 1^{\text {ctr1-1 }}$ seedlings grown on MS medium \pm ACC. Data represent the means and SD $(n>35) . \mathbf{k}$, CTR1-KD ${ }^{\text {ctr1-1 }}$ does not influence the stability of EIN2CEND. Arabidopsis mesophyll protoplasts were co-transfected with plasmid GFP-EIN2-CEND and an increasing amount of plasmid expressing GFP- $\Delta$ NT-CTR $1^{\text {ctr1-1 }}$ and incubated for $16 \mathrm{~h}$. Total protein extract was prepared from the transfected protoplasts and further analyzed by immunoblotting with anti-GFP antibody. Free GFP was used as a transfection control. I, CTR1 does not phosphorylate EBF2. In vitro kinase assay for purified $\triangle \mathrm{NT}$-CTR1 or $\triangle \mathrm{NT}$-CTR1 ${ }^{\text {ctr1-1 }}$ with the EIN2-CEND, EIN3, or EBF2. EIN2-CEND and EIN3 were used as positive and negative control, respectively. The indicated proteins were incubated together in kinase reaction buffer, separately by SDS/PAGE, and the incorporated radiolabel was detected by autoradiography. 


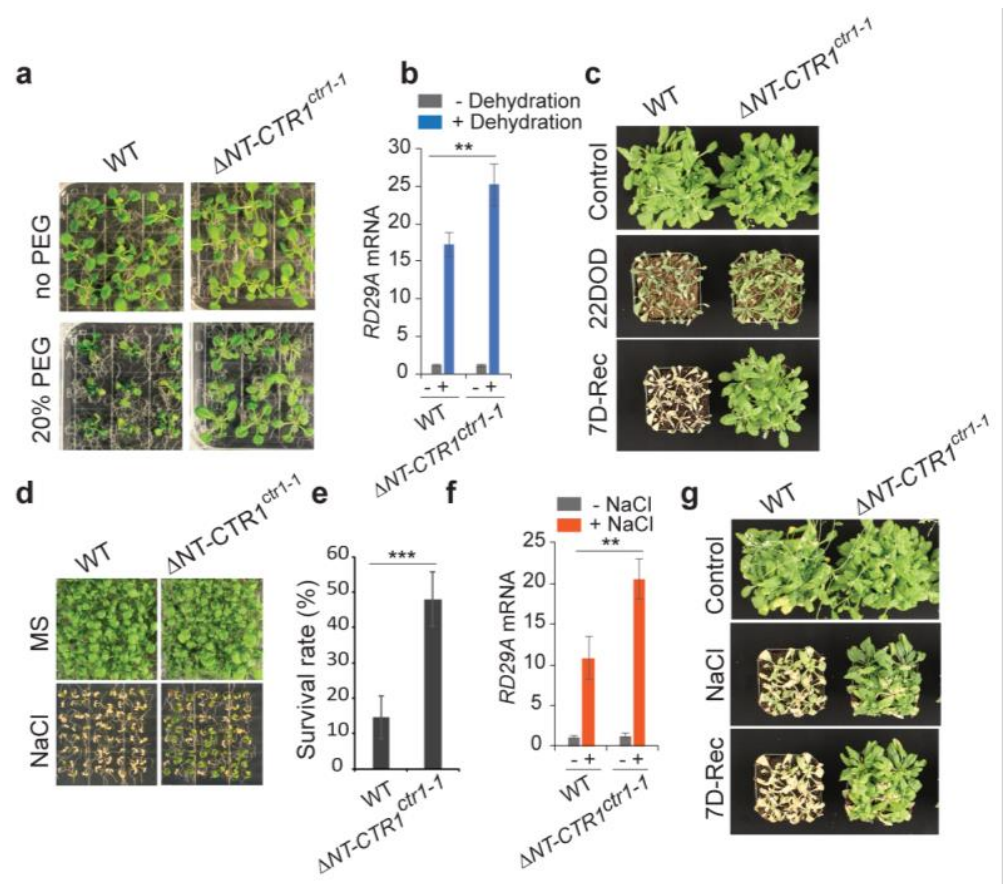

\section{Fig. 6. Increased CTR1 nuclear accumulation enhances plant's resilience to abiotic}

stress. a, One-week-old light-grown seedlings were transferred MS medium containing 20\% seedlings were placed on filter papers and dried for $3 \mathrm{~h}$ at room temperature and used for the analysis. Data represent the means and SD for three biological replicates, ${ }^{* *} p<0.01$, Student's t-test. c, Two-week-old WT and 35S:GFP- $\triangle N T-C T R 1^{\text {ctr1-1 }}$ seedlings were subjected to water stress by withdrawing water for 22-d (22 DOD), followed by 7-d of re-watering recovery (7DRec). d, Seedlings were grown on MS medium containing with or without $175 \mathrm{mM} \mathrm{NaCl}$ for 3weeks and the survival rate of the seedlings were counted. e, The quantification of survival rate of seedlings in (c), Data represent the means and SD for three biological replicates, ${ }^{\star * *} p<$ 0.001 , Student's $t$-test. f, Relative expression of RD29A gene. One-week-old seedlings were treated with $175 \mathrm{mM} \mathrm{NaCl}$ solution for $3 \mathrm{~h}$ and RNA was extracted. Data represent the means and SD for three biological replicates, ${ }^{* *} p<0.01$, Student's $t$-test. $\mathbf{g}$, Two-week-old seedlings were irrigated with $300 \mathrm{mM} \mathrm{NaCl}$ solution every 4 days for $28-\mathrm{d}$, then watered normally for 7 -d. 

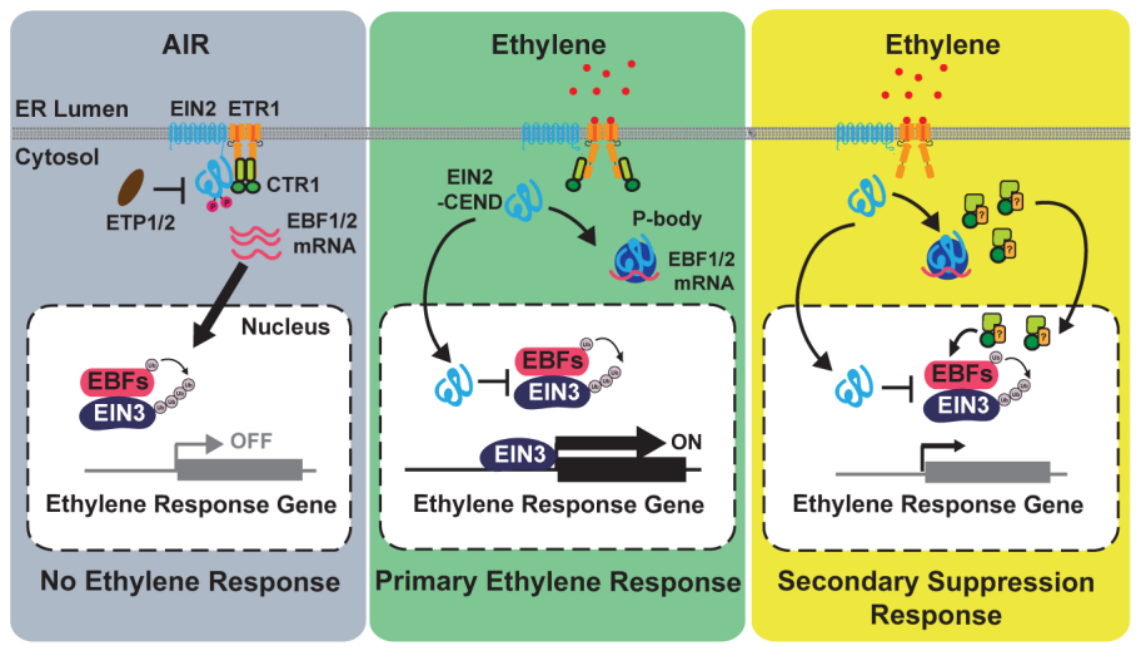

\section{Fig. 7}

Fig. 7. Model for ethylene-induced CTR1 nuclear translocation and suppression of

ethylene response. In the absence of ethylene (air), the ethylene receptors, CTR1, and EIN2 are mainly localized to the ER. The receptor-activated CTR1 phosphorylates EIN2, which leads to the proteolytic degradation of EIN2 via EIN2-TARGETING PROTEIN 1 (ETP1) and ETP2. In the presence of ethylene, the receptors, hence CTR1, are inactivated. Inactivated CTR1 no longer phosphorylates EIN2 and the C-terminal domain of EIN2 (EIN2-CEND) is proteolytically cleaved and translocates to the nucleus to initiate primary ethylene response via EIN3 activation. EIN2-CEND is also targeted to processing body (P-body) to suppress EBFs mRNA translation. After the nuclear translocation of EIN2-CEND, CTR1 is subsequently released from the receptors and re-locates to the nucleus via an unknown mechanism. The nuclear-localized CTR1 directly interacts with EBF2 resulting in promoting the degradation of EIN3, which ultimately counteracts EIN2-mediated activation of EIN3 to fine-tune ethylene responses. The yellow square with a question mark indicates an unknown cargo protein that delivers CTR1 to the nucleus. Arrows indicate the movement of ethylene signaling components or positive influence; blunted ends indicate inhibition. 


\section{References}

1. Abeles, F.B., Morgan, P.W. \& Saltveit, M.E.J. Ethylene in plant biology. San Diego, CA: Academic Press. (1992).

2. Bleecker, A.B. \& Kende, H. Ethylene: a gaseous signal molecule in plants. Annu. Rev. Cell. Dev. Biol. 16, 1-18 (2000).

3. Alonso, J.M. \& Ecker, J.R. The ethylene pathway: a paradigm for plant hormone signaling and interaction. Science's STKE : signal transduction knowledge environment 2001, re1 (2001).

4. Hattori, Y. et al. The ethylene response factors SNORKEL1 and SNORKEL2 allow rice to adapt to deep water. Nature 460, 1026-1030 (2009).

5. Lee, H.Y., Chen, Z., Zhang, C. \& Yoon, G.M. Editing of the OsACS locus alters phosphate deficiency-induced adaptive responses in rice seedlings. J. Exp. Bot. 70, 1927-1940 (2019).

6. Song, L. \& Liu, D. Ethylene and plant responses to phosphate deficiency. Frontiers in plant science 6, 796 (2015).

7. Thao, N.P. et al. Role of Ethylene and Its Cross Talk with Other Signaling Molecules in Plant Responses to Heavy Metal Stress. Plant Physiol. 169, 73-84 (2015).

8. Tao, J.J. et al. The Role of Ethylene in Plants Under Salinity Stress. Frontiers in plant science 6, 1059 (2015).

9. Chang, C. \& Stadler, R. Ethylene hormone receptor action in Arabidopsis. Bioessays 23, 619-627 (2001).

10. Guo, H. \& Ecker, J.R. The ethylene signaling pathway: new insights. Curr. Opin. Plant Biol. 7, 40-49 (2004). 
11. Kieber, J.J., Rothenberg, M., Roman, G., Feldmann, K.A. \& Ecker, J.R. CTR1, a negative regulator of the ethylene response pathway in Arabidopsis, encodes a member of the raf family of protein kinases. Cell 72, 427-441 (1993).

12. Alonso, J.M., Hirayama, T., Roman, G., Nourizadeh, S. \& Ecker, J.R. EIN2, a bifunctional transducer of ethylene and stress responses in Arabidopsis. Science (New York, N.Y.) 284, 2148-2152 (1999).

13. Bisson, M.M., Bleckmann, A., Allekotte, S. \& Groth, G. EIN2, the central regulator of ethylene signalling, is localized at the ER membrane where it interacts with the ethylene receptor ETR1. Biochem. J. 424, 1-6 (2009).

14. Clark, K.L., Larsen, P.B., Wang, X. \& Chang, C. Association of the Arabidopsis CTR1 Raf-like kinase with the ETR1 and ERS ethylene receptors. Proceedings of the National Academy of Sciences of the United States of America 95, 54015406 (1998).

15. Gao, Z. et al. Localization of the Raf-like kinase CTR1 to the endoplasmic reticulum of Arabidopsis through participation in ethylene receptor signaling complexes. J. Biol. Chem. 278, 34725-34732 (2003).

16. Ju, C. et al. CTR1 phosphorylates the central regulator EIN2 to control ethylene hormone signaling from the ER membrane to the nucleus in Arabidopsis. Proceedings of the National Academy of Sciences of the United States of America 109, 19486-19491 (2012).

17. Qiao, H. et al. Processing and subcellular trafficking of ER-tethered EIN2 control response to ethylene gas. Science (New York, N.Y.) 338, 390-393 (2012). 
18. Wen, X. et al. Activation of ethylene signaling is mediated by nuclear translocation of the cleaved EIN2 carboxyl terminus. Cell Res. 22, 1613-1616 (2012).

19. Merchante, C. et al. Gene-specific translation regulation mediated by the hormone-signaling molecule EIN2. Cell 163, 684-697 (2015).

20. Li, W. et al. EIN2-directed translational regulation of ethylene signaling in Arabidopsis. Cell 163, 670-683 (2015).

21. Binder, B.M. et al. Arabidopsis seedling growth response and recovery to ethylene. A kinetic analysis. Plant Physiol. 136, 2913-2920 (2004).

22. Binder, B.M. et al. The Arabidopsis EIN3 binding F-Box proteins EBF1 and EBF2 have distinct but overlapping roles in ethylene signaling. Plant Cell 19, 509-523 (2007).

23. Seo, D.H. \& Yoon, G.M. Light-induced stabilization of ACS contributes to hypocotyl elongation during the dark-to-light transition in Arabidopsis seedlings. Plant J. 98, 898-911 (2019).

24. Shakeel, S.N. et al. Ethylene Regulates Levels of Ethylene Receptor/CTR1 Signaling Complexes in Arabidopsis thaliana. J. Biol. Chem. 290, 12415-12424 (2015).

25. Huang, Y., Li, H., Hutchison, C.E., Laskey, J. \& Kieber, J.J. Biochemical and functional analysis of CTR1, a protein kinase that negatively regulates ethylene signaling in Arabidopsis. Plant J. 33, 221-233 (2003). 
26. Mayerhofer, H., Panneerselvam, S. \& Mueller-Dieckmann, J. Protein kinase domain of CTR1 from Arabidopsis thaliana promotes ethylene receptor cross talk. J. Mol. Biol. 415, 768-779 (2012).

27. Binder, B.M. Time-Lapse Imaging to Examine the Growth Kinetics of Arabidopsis Seedlings in Response to Ethylene. Methods in molecular biology (Clifton, N.J.) 1573, 211-222 (2017).

28. Binder, B.M., Mortimore, L.A., Stepanova, A.N., Ecker, J.R. \& Bleecker, A.B. Short-term growth responses to ethylene in Arabidopsis seedlings are EIN3/EIL1 independent. Plant Physiol. 136, 2921-2927 (2004).

29. Zhang, F. et al. EIN2 mediates direct regulation of histone acetylation in the ethylene response. Proceedings of the National Academy of Sciences of the United States of America 114, 10274-10279 (2017).

30. Nelson, B.K., Cai, X. \& Nebenfuhr, A. A multicolored set of in vivo organelle markers for co-localization studies in Arabidopsis and other plants. Plant J. 51, 1126-1136 (2007). 